\title{
Electroluminescent properties of erbium-doped III-N light-emitting diodes
}

\author{
J. M. Zavada ${ }^{\text {a) }}$ \\ U.S. Army Research Office, Durham, North Carolina 27709 \\ S. X. Jin, N. Nepal, J. Y. Lin, and H. X. Jiang \\ Department of Physics, Kansas State University, Manhattan, Kansas 66506 \\ P. Chow and B. Hertog \\ SVT Associates, Incorporated, Eden Prairie, Minnesota 55344
}

(Received 9 September 2003; accepted 18 December 2003)

\begin{abstract}
We report on the synthesis of Er-doped III-N double heterostructure light-emitting diodes (LEDs) and their electroluminescence (EL) properties. The device structures were grown through a combination of metalorganic chemical vapor deposition (MOCVD) and molecular-beam epitaxy (MBE) on $c$-plane sapphire substrates. The $\mathrm{AlGaN}$ layers, with an $\mathrm{Al}$ concentration of $\sim 12 \%$, were prepared by MOCVD and doped with $\mathrm{Si}$ or $\mathrm{Mg}$ to achieve $n$ - and $p$-type conductivity, respectively. The Er+O-doped GaN active region was grown by $\mathrm{MBE}$ and had a thickness of $50 \mathrm{~nm}$. The $\mathrm{Er}$ concentration was estimated to be $\sim 10^{18} \mathrm{~cm}^{-3}$. The multilayer $n$-AlGaN/GaN:Er/ $p$-AlGaN structures were processed into LEDs using standard etching and contacting methods. Several different LEDs were produced and EL spectra were recorded with both forward and reverse bias conditions. Typically, the EL under reverse bias was five to ten times more intense than that under forward bias. The LEDs displayed a number of narrow emission lines representative of the GaN:Er system (green: $539 \mathrm{~nm}, 559 \mathrm{~nm}$; infrared: $1000 \mathrm{~nm}, 1530 \mathrm{~nm}$ ). While some current crowding was observed, green emission was visible under ambient room conditions at $300 \mathrm{~K}$. At cryogenic temperatures, the emission lines increased in intensity and had a narrower linewidth. EL spectra were recorded down to $10 \mathrm{~K}$ and the L-I characteristics were systematically measured. The power output of the brightest LEDs was approximately $2.5 \mathrm{~W} / \mathrm{m}^{2}$ at $300 \mathrm{~K}$. (c) 2004 American Institute of Physics. [DOI: 10.1063/1.1647271]
\end{abstract}

Beginning with the work of Ennen et al., ${ }^{1}$ study of the optical properties of III-V semiconductors doped with rareearth (RE) elements has received considerable attention. ${ }^{2}$ Due to the importance of the $1.54 \mu \mathrm{m}$ region for optical communications, Er has been the main RE element to be investigated in these semiconductors. Since the roomtemperature intensity of the light emission is strongly dependent upon the band-gap energy of the host material, ${ }^{3}$ wide band-gap semiconductors, such as III-V nitride semiconductors, appear to be excellent materials for device applications. RE-doped wide gap semiconductors offer the prospect of temperature-stable electrically pumped optical amplifiers and electroluminescent light emitters operating at wavelengths from the visible to the infrared. Er-doped light-emitting diodes (LEDs) represent a class of devices that emit light from the intra- $4 f$ transitions of the $\mathrm{Er}^{3+}$ ions by means of electrical injection. Under forward bias, energy from electron-hole pairs can be transferred to the $\mathrm{Er}^{3+}$ ions leading to the excitation of the $4 f$ electrons. This process likely involves an intermediate defect level in the $\mathrm{GaN}$ host. The $\mathrm{Er}^{3+}$ ions can also be directly excited by impact of hot carriers under reverse bias. ${ }^{4}$ This process is similar to excitation under cathodoluminescence. In both cases, the intra- $4 f$ transitions of $\mathrm{Er}^{3+}$ ions give rise to sharp emission lines whose wavelengths are largely independent of the host material. This wavelength stability occurs because the outer $5 s$ and $5 p$ electron shells are filled and the inner $4 f$ shell electrons are screened from interactions with the host. In wide band-gap

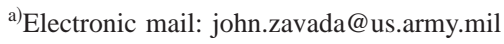

semiconductors, emission from the $\mathrm{Er}^{3+}$ ions is also very stable with respect to temperature of the host. Energy backtransfer and Auger processes are significantly reduced in such materials and luminescence from $\mathrm{Er}^{3+}$ ions can be observed at temperatures up to $\sim 500 \mathrm{~K}^{5,6}$

Qiu et al. ${ }^{7}$ demonstrated room temperature operation of an electroluminescent device (ELD) emitting at $1.54 \mu \mathrm{m}$ based on an Er-doped GaN semiconductor film. The device consisted of a metal $/ i-\mathrm{GaN} / n-\mathrm{GaN}$ structure grown by chemical vapor deposition (CVD) on sapphire. The active region of the device, the $i$-GaN layer, was coimplanted with $\mathrm{Er}^{+}$and $\mathrm{O}^{+}$ions. High-field injection of electrons under reverse bias produced IR emission at $\sim 1.54 \mu \mathrm{m}$ associated with the ${ }^{4} I_{13 / 2} \rightarrow{ }^{4} I_{15 / 2}$ transition of $\mathrm{Er}^{3+}$ ions. No $\mathrm{Er}^{3+}$-related electroluminescence (EL) was observed under forward bias conditions. Steckl et al. achieved a variety of RE-doped GaN ELDs grown by molecular-beam epitaxy (MBE) using solid sources for Ga and the REs. Initial results were obtained with Er-doped $\mathrm{GaN}$ films, ${ }^{8}$ and subsequently extended to Pr-, Eu-, and Tm-doped films. ${ }^{9}$ Emission spectra from the GaN:Er ELDs displayed IR emission at $\sim 1.5 \mu \mathrm{m}$. The spectra were measured over a temperature range from 250 to $400 \mathrm{~K}$, with little change in the emission intensity spectrum. The ELDs also produced green emission peaks at $\sim 537$ and $558 \mathrm{~nm}$, corresponding to radiative transitions from the ${ }^{2} H_{11 / 2} \rightarrow{ }^{4} I_{15 / 2}$ and ${ }^{4} S_{3 / 2} \rightarrow{ }^{4} I_{15 / 2}$ states, respectively. Using MBE methods, $\mathrm{Ng}$ et al. ${ }^{10}$ produced similar GaN:Er ELDs and observed both green and infrared emission. Morishima et al. ${ }^{11}$ used gas-source MBE growth with a metallic source for Eu to produce metal-insulator-semiconductor di- 


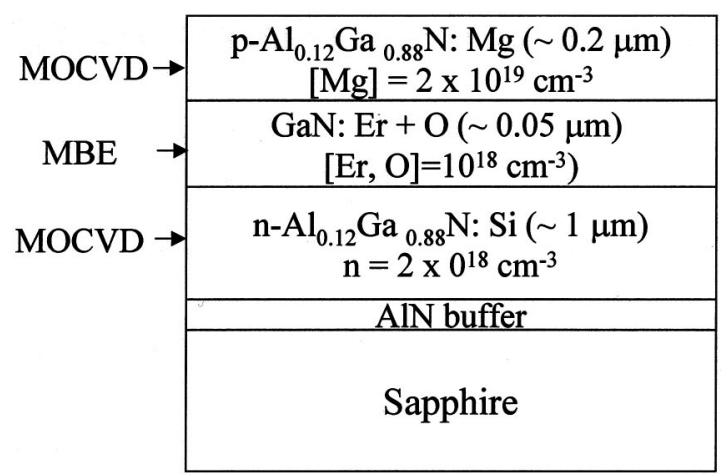

(a)

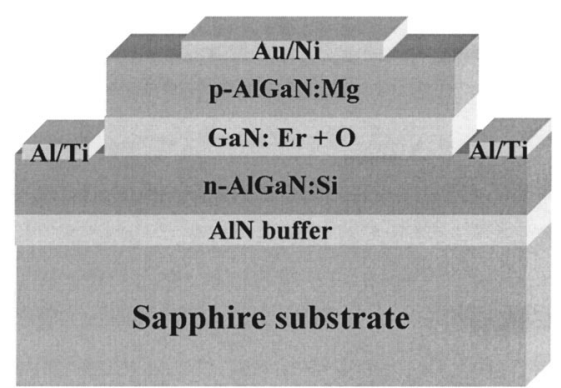

(b)

FIG. 1. (a) Schematic diagram of the III-N DH grown by MOCVD and MBE. (b) Schematic drawing of the processed LED.

odes on $n$-type Si (111) substrates. The EL spectrum from the $\mathrm{GaN}: \mathrm{Eu}$ device, operated under a reverse dc bias of $\sim 5 \mathrm{~V}$, had a prominent peak at $\sim 622 \mathrm{~nm}$, corresponding to a radiative transition from the ${ }^{5} D_{0} \rightarrow{ }^{7} F_{2}$ states.

In this letter, we report on the synthesis of Er-doped III-N LEDs based on AlGaN/GaN double heterostructures (DHs) and their EL properties. The DHs were produced through a combination of metalorganic CVD (MOCVD) and MBE methods. At $300 \mathrm{~K}$, these devices exhibited EL spectra characteristic of the visible and IR emission peaks of $\mathrm{Er}^{3+}$ ions under both forward and reverse biases. This is a demonstration of Er-doped $p-n$ diodes formed in the III-N material system.

Synthesis of the nitride DHs involved a multistage growth process. First, AlGaN epilayers were grown by MOCVD on sapphire (0001) substrates, as described elsewhere. ${ }^{12}$ The $n$-type layers were $\sim 1 \mu \mathrm{m}$ thick, with an $\mathrm{Al}$ content $\sim 12 \%$, and a Si doping $\sim 2 \times 10^{18} \mathrm{~cm}^{-3}$. The $\mathrm{Al}_{0.12} \mathrm{Ga}_{0.88} \mathrm{~N}$ alloy has a band gap of $\sim 3.75 \mathrm{eV}$. Subsequently, the GaN: Er epilayer was grown by MBE with an $\mathrm{Er}$ concentration of $\sim 10^{18} \mathrm{~cm}^{-3}$. ${ }^{13}$ This layer was codoped with $\mathrm{O}$ to about the same concentration and had a thickness of $50 \mathrm{~nm}$. After this stage, a second growth of AlGaN by MOCVD was performed. The top $p$-type AlGaN layer was $\sim 200 \mathrm{~nm}$ thick, again with $\sim 12 \% \mathrm{Al}$, and with $\mathrm{Mg}$ doping $\sim 2 \times 10^{19} \mathrm{~cm}^{-3}$. Room-temperature hole concentration was $\sim 10^{17} \mathrm{~cm}^{-3}$. A schematic of the DH is shown in Fig. 1(a). Prior studies have shown that this growth sequence does not alter the photoluminescence (PL) properties of the $\mathrm{GaN}: \mathrm{Er}$ epilayer. ${ }^{14}$

The fabrication procedures for Er-doped GaN LEDs were very similar to those of III-N blue and UV LEDs. ${ }^{15,16}$ The devices were patterned by photolithography and inductively coupled plasma dry etching. Bilayers of $\mathrm{Ni}(20$ peak IR EL intensity measured at $1 \mu \mathrm{m}$ and $1.54 \mu \mathrm{m}$.
Downloaded 30 Mar 2011 to 129.118.237.235. Redistribution subject to AIP license or copyright; see http://apl.aip.org/about/rights_and_permissions

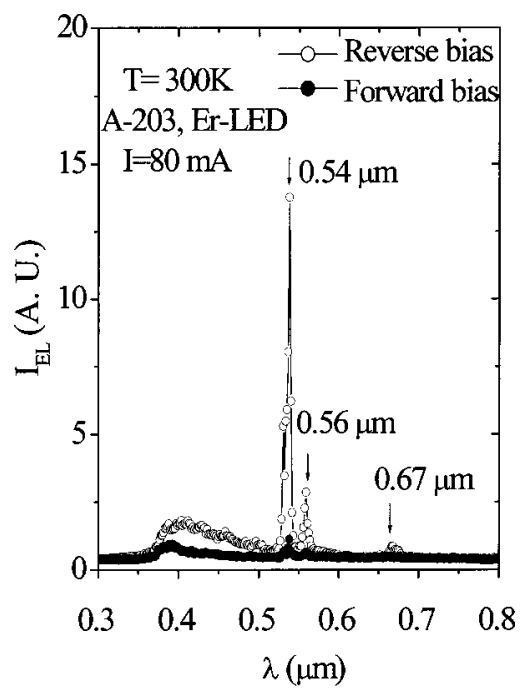

FIG. 2. Visible EL spectra for the Er-doped GaN LED with an active layer thickness of $50 \mathrm{~nm}$ under reverse bias (open circles) and under forward bias (solid dots).

$\mathrm{nm}) / \mathrm{Au}(200 \mathrm{~nm})$ and Al $(300 \mathrm{~nm}) / \mathrm{Ti}(20 \mathrm{~nm})$ were deposited by electron-beam evaporation as $p$ - and $n$-type ohmic contacts. The contacts were thermally annealed in a nitrogen ambient at $650{ }^{\circ} \mathrm{C}$ for $5 \mathrm{~min}$, which was below the MBE growth temperature, and so the diffusion or intermixing of the epilayers was not expected. Figure 1(b) is a schematic diagram of the processed Er-doped III-N LED. The power output was measured from the sapphire substrate side of unpackaged bare chips by a wafer probe. Both the power output and EL spectra were measured under dc bias conditions.

Figure 2 shows the visible EL spectra, measured at 300 $\mathrm{K}$, from a typical GaN:Er DH LED produced in these experiments. Measurements were made under reverse and forward biases using a current of $80 \mathrm{~mA}$. The spectra display the main emission peaks of $\mathrm{Er}^{3+}$ ions. There is also a broad emission band from $\sim 375 \mathrm{~nm}-500 \mathrm{~nm}$, but no GaN bandedge emission was observed. While the EL spectra are simi-
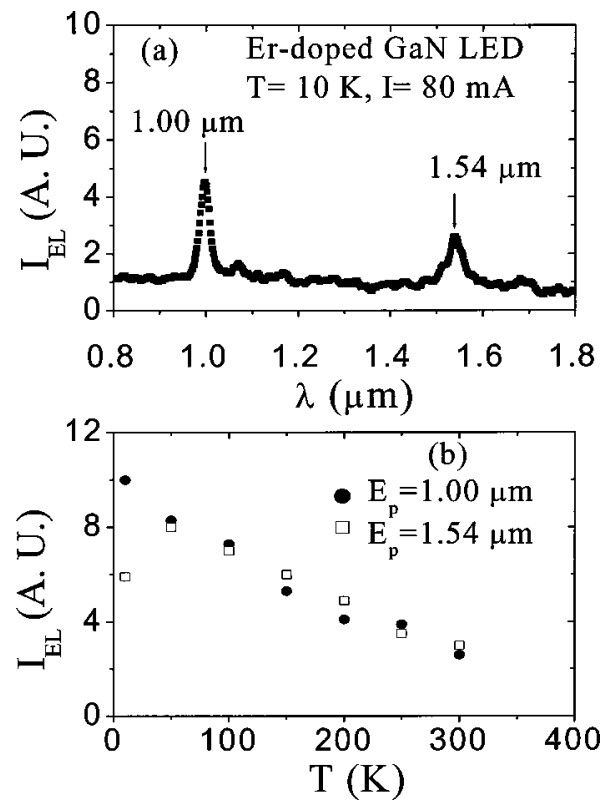

FIG. 3. (a) IR EL spectra at $10 \mathrm{~K}$ for the Er-doped III-N LED under reverse bias with a driving current of $80 \mathrm{~mA}$. (b) The temperature dependence of the peak IR EL intensity measured at $1 \mu \mathrm{m}$ and $1.54 \mu \mathrm{m}$. 

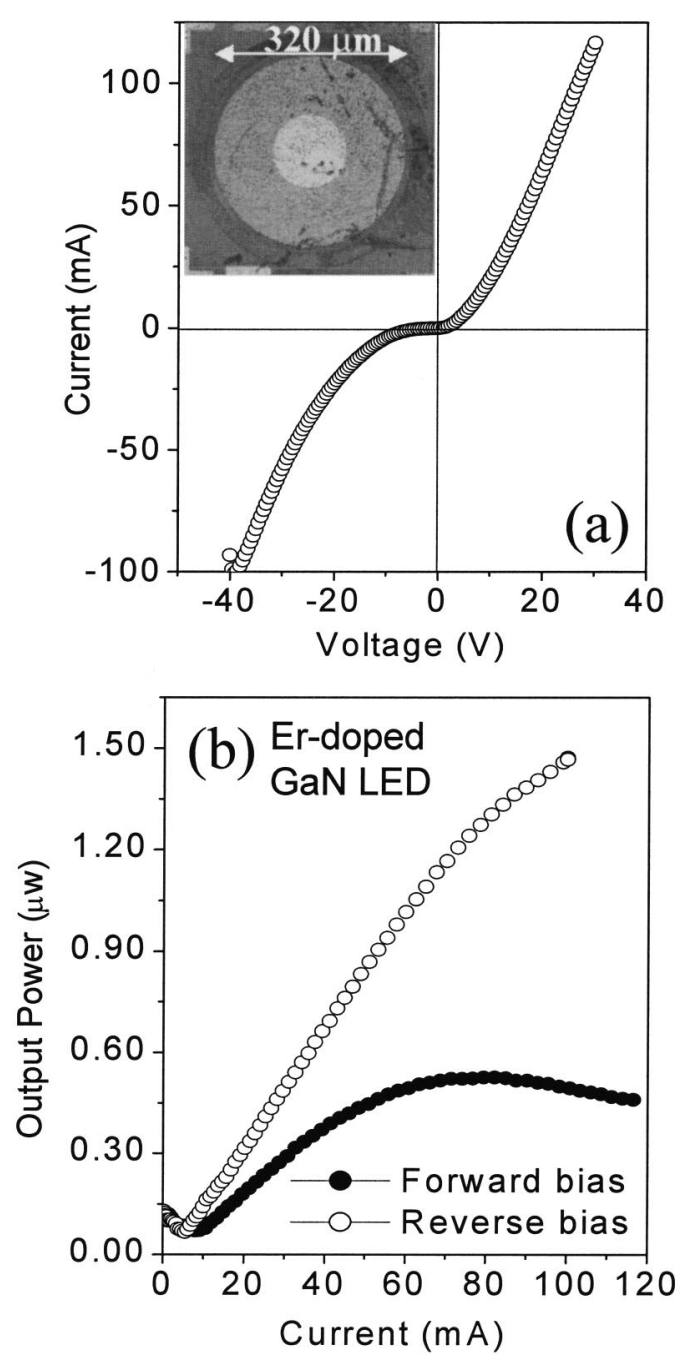

FIG. 4. (a) $I-V$ characteristics at $300 \mathrm{~K}$ of the Er-doped GaN LED, the inset shows an optical micrograph of the LED; (b) Output power, integrated over the visible spectrum, under reverse bias (open circles) and under forward bias (solid dots) conditions.

lar, the emission lines are ten to fifteen times less intense with forward biasing. This is in agreement with other studies on Er-doped LEDs based on $\mathrm{Si}$ and $\mathrm{SiO}_{x}$ materials. ${ }^{4,17}$ However, using an Er-doped GaAs/InGaP DH LED, Koizumi et al. ${ }^{18}$ found EL only under reverse bias conditions. Apparently, under reverse bias, the $\mathrm{Er}^{3+}$ ions are excited by hot electrons from the junction. While the excitation mechanism under forward bias is not fully understood, it is assumed to be due to energy transfer from electron-hole pairs through an intermediate state to the $\mathrm{Er}^{3+}$ ions. Consequently, a DH should be more effective in confining electron-hole recombination to the region in which the $\mathrm{Er}^{3+}$ ions are situated.

Figure 3(a) shows the low-temperature (10 K) EL spectrum measured in the IR region under reverse bias. Two lines at $1000 \mathrm{~nm}$ and $1540 \mathrm{~nm}$ are prominent. The temperature dependence of the peak EL intensity of these IR lines is shown in Fig. 3(b). As found in previous studies, the peak EL intensity of these lines is inversely proportional to the ambient temperature. ${ }^{5}$ The observed EL decrease of $\sim 38 \%$ from $10 \mathrm{~K}$ to $300 \mathrm{~K}$ is slightly greater than the reported PL decrease in Er-doped GaN.

In Fig. 4(a), current-voltage $(I-V)$ characteristics are displayed for one of the DH LEDs having a diameter of $320 \quad$ Takeda, Jpn. J. Appl. Phys., Part 1 42, 2223 (2003).
Downloaded 30 Mar 2011 to 129.118.237.235. Redistribution subject to AIP license or copyright; see http://apl.aip.org/about/rights_and_permissions nm. Compared with III-N blue and UV LEDs, the rectification behavior is weaker for the Er-doped LED. The L-I plots (integrated optical power output versus forward and reverse bias) are shown in Fig. 4(b). Turn-on voltage was $\sim 3.5 \mathrm{~V}$ for forward bias and $\sim-4.5 \mathrm{~V}$ for reverse bias conditions. Under forward bias, the EL intensity initially increases with increasing drive current, but then saturates and reaches a maximum at $\sim 70 \mathrm{~mA}$. The saturation of the intensity may indicate that the concentration of optically active Er centers is a limiting factor for the EL intensity under the forward bias. Nonradiative processes may also be involved. Under reverse bias, the output power continues to increase even above $70 \mathrm{~mA}$. At $100 \mathrm{~mA}$, the integrated optical power output under reverse bias is nearly three times that under forward bias. Based on the dimensions of the LED, it is estimated that the maximum output power is approximately 2.5 $\mathrm{W} / \mathrm{m}^{2}$ at $300 \mathrm{~K}$.

In summary, we have demonstrated EL emission from Er-doped GaN LEDs that were grown by MOCVD and MBE. Their EL properties were investigated under forward and reverse bias conditions. The EL spectra at room temperature indicated effective excitation of the $\mathrm{Er}^{3+}$ ions by current injection. The EL intensity of all emission lines was ten to fifteen times higher under reverse bias than under forward bias. The output power, integrated over the visible spectrum, of the DH LED was estimated to be $\sim 2.5 \mathrm{~W} / \mathrm{m}^{2}$ at $300 \mathrm{~K}$.

The authors at KSU wish to acknowledge support by the ARO. Work done at SVT Associates was partially funded by MDA Contract No. F49620-02-C-0069, Dr. Gernot Pomrenke AFOSR, technical monitor.

${ }^{1}$ H. Ennen, J. Schneider, G. Pomrenke, and A. Axmann, Appl. Phys. Lett. 43, 943 (1983).

2 Mater. Res. Soc. Symp. Proc. , 301 (1993); ibid. Mater. Res. Soc. Symp. Proc., 422 (1996); Mater. Sci. Eng., B 81, (2001). Jpn. J. Appl. Phys., Part 2 29, L524 (1990).

${ }^{4}$ S. Lombardo, S. U. Campisano, G. N. van den Hoven, and A. Polman, J. Appl. Phys. 77, 6504 (1995).

${ }^{5}$ M. Thaik, U. Hömmerich, R. N. Schwartz, R. G. Wilson, and J. M. Zavada, Appl. Phys. Lett. 71, 2641 (1997).

${ }^{6}$ W. J. Choyke, R. P. Devaty, L. I. Clemen, M. Yoganathan, G. Pensl, and C. Hassler, Appl. Phys. Lett. 65, 1668 (1994).

${ }^{7}$ C. H. Qiu, M. W. Leksono, J. I. Pankove, J. T. Torvik, R. J. Feuerstein, and F. Namavar, Appl. Phys. 66, 562 (1995).

${ }^{8}$ A. J. Steckl and R. Birkhahn, Appl. Phys. Lett. 73, 1700 (1998).

${ }^{9}$ A. J. Steckl, J. Heikenfeld, M. Garter, R. Birkhahn, and D. S. Lee, Compound Semicond. 48, 6 (2000).

${ }^{10} \mathrm{H}$. M. Ng, State-of-the-Art Program on Compound Semiconductors XXXVI and Wide Band-gap Semiconductors for Photonic and Electronic Devices and Sensors II (Electrochemical Society, New York, 2002), Vol. 2002-3.

${ }^{11}$ S. Morishima, T. Maruyama, M. Tanaka, Y. Masumoto, and K. Akimoto, Phys. Status Solidi A 176, 113 (1999).

${ }^{12}$ K. B. Nam, J. Li, M. L. Nakarmi, J. Y. Lin, and H. X. Jiang, Appl. Phys. Lett. 81, 1038 (2002).

${ }^{13}$ SVT Associates, Inc., Eden Prarie, MN (unpublished).

${ }^{14}$ J. M. Zavada, J. Y. Lin, H. X. Jiang, P. Chow, B. Hertog, U. Hömmerich, E. E. Nyein, and H. A. Jenkinson, Mater. Sci. Eng. B 105, 118 (2003).

${ }^{15}$ S. X. Jin, J. Li, J. Shakya, J. Y. Lin, and H. X. Jiang, Appl. Phys. Lett. 78, 3532 (2001).

${ }^{16}$ K. H. Kim, J. Li, S. X. Jin, J. Y. Lin, and H. X. Jiang, Appl. Phys. Lett. 83, 566 (2003).

${ }^{17}$ S. Coffa, G. Franzo, and F. Priolo, Mater. Res. Bull. 23, 25 (1998).

${ }^{18}$ A. Koizumi, Y. Fujiwara, K. Inoue, A. Urakami, T. Yoshikane, and Y. Takeda, Jpn. J. Appl. Phys., Part 1 42, 2223 (2003).

cense or copyright; see http://apl.aip.org/about/rights_and_permissions
${ }^{3}$ P. N. Favennec, H. L'Haridon, D. Moutonnet, M. Salvi, and M. Gauneau, 